\title{
Amplification and nonlinear mechanisms in plane Couette flow
}

\author{
Dennice F. Gayme, ${ }^{1}$ Beverley J. McKeon, ${ }^{1}$ Bassam Bamieh, ${ }^{2}$ Antonis Papachristodoulou, ${ }^{3}$ \\ and John C. Doyle ${ }^{3}$ \\ ${ }^{1}$ Division of Engineering and Applied Science, California Institute of Technology, Pasadena, \\ California 91125, USA \\ ${ }^{2}$ Department of Mechanical Engineering, University of California at Santa Barbara, Santa Barbara, \\ California 93106, USA \\ ${ }^{3}$ Department of Engineering Science, University of Oxford, Parks Road, Oxford OX1 3PJ, United Kingdom
}

(Received 18 November 2010; accepted 17 May 2011; published online 17 June 2011)

\begin{abstract}
We study the input-output response of a streamwise constant projection of the Navier-Stokes equations for plane Couette flow, the so-called 2D/3C model. Study of a streamwise constant model is motivated by numerical and experimental observations that suggest the prevalence and importance of streamwise and quasi-streamwise elongated structures. Periodic spanwise/wall-normal $(z-y)$ plane stream functions are used as input to develop a forced $2 \mathrm{D} / 3 \mathrm{C}$ streamwise velocity field that is qualitatively similar to a fully turbulent spatial field of direct numerical simulation data. The inputoutput response associated with the $2 \mathrm{D} / 3 \mathrm{C}$ nonlinear coupling is used to estimate the energy optimal spanwise wavelength over a range of Reynolds numbers. The results of the input-output analysis agree with previous studies of the linearized Navier-Stokes equations. The optimal energy corresponds to minimal nonlinear coupling. On the other hand, the nature of the forced $2 \mathrm{D} / 3 \mathrm{C}$ streamwise velocity field provides evidence that the nonlinear coupling in the $2 \mathrm{D} / 3 \mathrm{C}$ model is responsible for creating the well known characteristic "S" shaped turbulent velocity profile. This indicates that there is an important tradeoff between energy amplification, which is primarily linear, and the seemingly nonlinear momentum transfer mechanism that produces a turbulent-like mean profile. (C) 2011 American Institute of Physics. [doi:10.1063/1.3599701]
\end{abstract}

\section{INTRODUCTION}

We study the input-output response of the Navier-Stokes (NS) equations for plane Couette flow. Although this type of analysis can be performed for a variety of input/output combinations for the full nonlinear equations, the mathematical complexity of such an endeavor makes it difficult to both obtain and interpret the results. Instead, we perform the analysis in the simplified setting of a nonlinear streamwise constant projection of the NS equations.

The choice of a streamwise constant model is motivated by studies of the linearized Navier-Stokes (LNS) equations, which show that streamwise constant features are the dominant mode shapes that develop under various perturbations about both the laminar ${ }^{1-4}$ and turbulent mean velocity ${ }^{5,6}$ profiles. In addition, streaks of streamwise velocity naturally arise from the set of initial conditions that produce the largest energy growth, ${ }^{7,8}$ namely, streamwise vortices. Even in linearly unstable flows, studies have shown that the amplitude of streamwise constant structures can exceed that of the linearly unstable modes. ${ }^{9,10}$ Bamieh and Dahleh ${ }^{2}$ explicitly showed that streamwise constant perturbations produce energy growth on the order of $R^{3}$ whereas disturbances with streamwise variations produce growth on the order of $R^{\frac{3}{2}}$.

The prevalence of large-scale streamwise constant structures is also supported by direct numerical simulation (DNS) and experiments. In Couette flow, DNS has long produced turbulent flows with very-large-scale streamwise and quasistreamwise structures in the core. ${ }^{11,12}$ Experimental high
Reynolds number studies have similarly identified largescale streamwise coherence in other flow configurations. ${ }^{13-16}$ At high Reynolds numbers (e.g., $R e_{\tau}>7300$ ), there is experimental evidence from pipes and turbulent boundary layers suggesting that these structures contain more energy than those nearer to the wall. ${ }^{14,16,17}$ The near-wall features, which also exhibit streamwise and quasi-streamwise alignment, are known to play a key role in energy production through the well studied "near-wall autonomous cycle."18-21

The dominance of streamwise constant features was previously used to motivate the study of a streamwise constant model for plane Couette flow. ${ }^{22}$ A stochastically forced version of this streamwise constant projection of NS was shown to reproduce important features of fully developed turbulence, including the shape of the turbulent velocity profile. Further, using Taylor's hypothesis, the same model also generated large-scale streaky structures that closely resemble large-scale features in the core. ${ }^{23}$ Given that maximum amplification of the LNS also occurs for the $k_{x}=0$ modes (i.e., in a streamwise constant sense), the analysis of a streamwise constant model can be viewed as a study of the full system along the direction of maximum amplification. In the present work, we use this approach to examine the interaction between the well studied linear amplification mechanisms and additional effects due to the nonlinear coupling in the streamwise constant model.

This paper is organized as follows. In Sec. II, we describe the streamwise constant (so-called 2D/3C) model and the idealized steady-state stream function model 
representing the cross-stream components of streamwise homogenous features. This stream function is used as input to a steady-state $2 \mathrm{D} / 3 \mathrm{C}$ streamwise momentum equation. The solution corresponding to each stream function input can be thought of as a forced solution of the respective streamwise deviation from the laminar flow. These streamwise velocity fields are compared to a spatial field of DNS data, obtained from the Kawamura group, ${ }^{24}$ in order to verify the ability of the model to capture relevant features of turbulent flow. We compute the spanwise/wall-normal $(z-y)$ plane forcing required to produce each of the stream functions described above and study the input-output response from this forcing input to the streamwise velocity's deviation from laminar. The optimal spanwise wavelength computed in this manner is consistent with linear studies. However, the nonlinearity in the model gives additional insight to the relationship between amplification and the turbulent velocity profile. In fact, this work demonstrates that there is an important tradeoff between linear amplification mechanisms and the nonlinearity required to develop an appropriately shaped turbulent velocity profile. The paper concludes with a summary of our results and directions for future work.

\section{MODELS}

\section{A. The $2 \mathrm{D} / 3 \mathrm{C}$ model}

The 2D/3C model for plane Couette flow discussed herein is obtained by setting the streamwise ( $x$-direction) velocity derivatives in the full NS equations to zero. ${ }^{25}$ This can be thought of as a projection of the NS equations into the streamwise constant space. The velocity field is then decomposed into components $\overrightarrow{\mathbf{u}}=\left[U+u_{s w}^{\prime}, V+v_{s w}^{\prime}, W+w_{s w}^{\prime}\right]$, where $(U, V, W)$ with $U=U(y)=y, V=W=0$ is the laminar flow, and $\left(u_{s w}^{\prime}, v_{s w}^{\prime}, w_{s w}^{\prime}\right)$ are the streamwise, wall-normal, and spanwise time dependent deviations, respectively, from laminar in the streamwise constant sense. One can explicitly show that, for Couette flow, this $2 \mathrm{D} / 3 \mathrm{C}$ formulation also results in a system with zero streamwise pressure gradient.

A stream function $\psi(y, z, t)$, such that

$$
v_{s w}^{\prime}=\frac{\partial \psi}{\partial z} ; \quad w_{s w}^{\prime}=-\frac{\partial \psi}{\partial y},
$$

ensures that the resulting model satisfies the appropriate $2 D$ continuity equation. This yields

$$
\begin{gathered}
\frac{\partial u_{s w}^{\prime}}{\partial t}=-\frac{\partial \psi}{\partial z} \frac{\partial u_{s w}^{\prime}}{\partial y}-\frac{\partial \psi}{\partial z} \frac{\partial U}{\partial y}+\frac{\partial \psi}{\partial y} \frac{\partial u_{s w}^{\prime}}{\partial z}+\frac{\Delta}{R} u_{s w}^{\prime} \\
\frac{\partial \Delta \psi}{\partial t}=-\frac{\partial \psi}{\partial z} \frac{\partial \Delta \psi}{\partial y}+\frac{\partial \psi}{\partial y} \frac{\partial \Delta \psi}{\partial z}+\frac{1}{R} \Delta^{2} \psi
\end{gathered}
$$

where $\Delta=\frac{\partial^{2}}{\partial y^{2}}+\frac{\partial^{2}}{\partial z^{2}}$. There is no slip or penetration at the wall, and periodic boundary conditions are assumed for the spanwise direction.

The Reynolds number employed for all computations described herein is $R=R_{w}=\frac{U_{w} h}{\nu}$, where the $U_{w}$ is the velocity of the top plate, $h$ is the channel height, and $\nu$ is the kinematic viscosity of the fluid. All distances and velocities are normalized by $h$ and $U_{w}$, respectively.

\section{B. The stream function model}

As a first step, we focus on the effect of large-scale streamwise elongated features in the core of a fully turbulent flow. We limit our study to cross-stream (i.e., wall-normal/ spanwise plane) inputs because energy amplification of perturbations (forcing) from the wall-normal and spanwise directions have been shown to scale as $R^{3}$ whereas all of the other input-output combinations admit only $R$ scaling. ${ }^{4} \mathrm{We}$ focus on the effect of cross-stream inputs, specifically those that represent the large-scale channel spanning streamwise structures that dominate the flow, ${ }^{26}$ on the streamwise component of the flow.

We are interested in developing a simple analytic model for the steady-state stream functions $\psi_{s s}(y, z)$ that will define our inputs. This will lead to computational tractability and better lends itself to analytical studies. In order to develop a phenomenologically accurate model, we examined the crossstream field $\psi_{s s}(y, z)$ created by integrating the streamwise averaged spanwise and wall-normal components of a fully turbulent three dimensional field of DNS data. ${ }^{24}$ This averaging gives us an approximation of a streamwise constant flow field. In the sequel, we refer to this streamwise averaged DNS field as the $x$-averaged DNS data. A full discussion of this velocity field and its use as an approximation for streamwise constant data (including an examination of the effects of the streamwise extent that it is being averaged over) is given in Refs. 22 and 27.

Our model development was further guided by the work of Barkley and Tuckerman, ${ }^{28}$ who showed that laminar-turbulent flow patterns in plane Couette flow can be reproduced using a cross-stream stream function of the form $\psi(y, z)=\psi_{0}(y)+\psi_{1}(y) \cos \left(k_{z} z\right)+\psi_{2}(y) \sin \left(k_{z} z\right)$. After confirming that their model is consistent with our DNS based estimate, we adopt their basic model with the zeroth-order term set to zero. A nonzero $\psi_{0}$ produces a nonzero-mean spanwise flow $w_{s s}^{\prime}$, which is not representative of the velocity field we are interested in studying. Combining their ideas with the information obtained from the DNS data led to the following simple doubly harmonic function

$$
\psi_{\mathrm{ss}}(y, z)=\varepsilon \sin ^{2}(q \pi y) \cos \left(\frac{2 \pi}{\lambda_{z}} z\right),
$$

which obeys the boundary conditions. ${ }^{22,27}$ Based on Eq. (2), the geometry and associated wall-normal extent of the structures are fixed. Thus, the parameter $\varepsilon$ dictates the intensity of the features, while $q$ indicates the wall-normal mode that we are considering.

In order to estimate the values for $\lambda_{z}, \varepsilon$, and $q$, we examine the $x$-averaged spanwise and wall-normal DNS fields. We verify that not only the $\psi_{s s}$ field but also the corresponding wall-normal and spanwise velocity fields based on differentiation of our model have the correct features. Figure 1(b) shows $\psi=-\frac{\partial w_{x a v e}^{\prime}}{\partial y}$ computed by integrating the $x$-averaged spanwise velocity $\left(w_{x_{\text {ave }}}^{\prime}(y, z)\right)$ from the DNS data at $R=3000$. The $\psi_{s s}$ model corresponding to $\mathrm{Eq}$. (2) with $q=1, \varepsilon=0.00675$ selected to match the magnitude of the integrated $w_{x_{\text {ave }}}^{\prime}$ and $v_{x_{\text {ave }}}^{\prime}$ DNS fields, and $\lambda_{z} \approx 1.8$ selected to match the fundamental spanwise wavelength of DNS is 


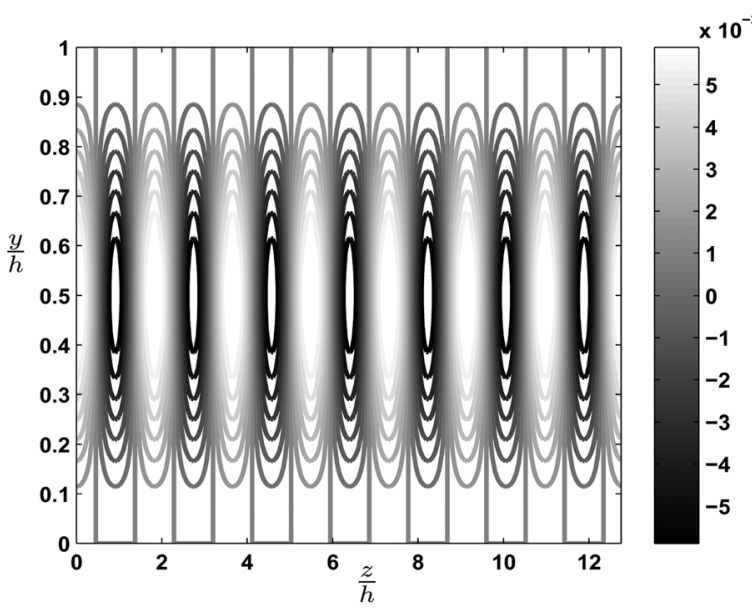

(a)

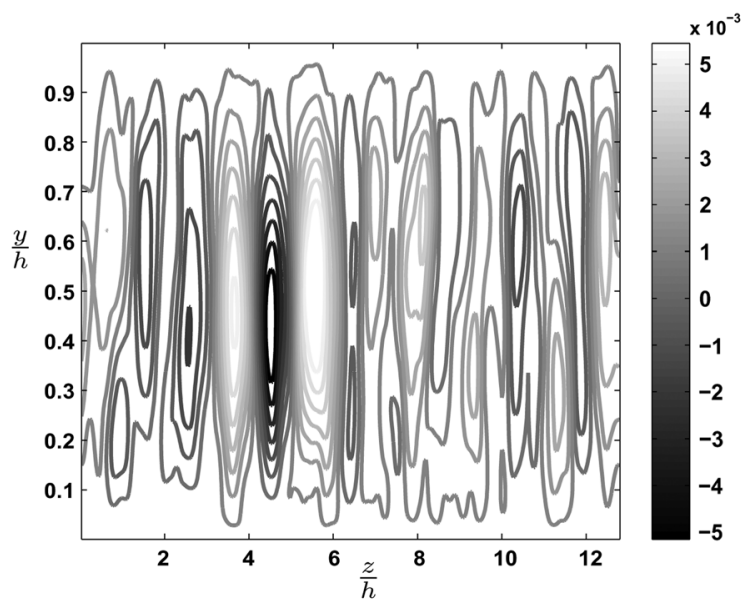

(b)

FIG. 1. (a) Contour plot of the first $y$ harmonic $(q=1)$ for the stream function model. This model represents the streamwise constant streaks and vortices commonly observed in DNS and experiments. (b) The stream function computed based on the $x$-averaged spanwise DNS velocity field, which was integrated to obtain the stream function, i.e., $\psi_{x_{\text {ave }}}(y, z)=-\frac{\partial w_{\text {xave }}^{\prime}}{\partial y}$. These plots are reproduced from Ref. 22.

provided in Figure 1(a). Comparison of Figures 1(b) and 1(a) indicate that the $\psi_{s s}$ model shows good agreement with the DNS data approximation in the region of highest signal. The region of highest signal is of primary interest because, with $q=1$, we are only looking at the first harmonic in both the wall-normal and spanwise directions.

\section{FORCED SOLUTIONS}

The steady-state solutions of Eq. (1a) corresponding to steady-state stream functions of the form of Eq. (2) are of interest for two reasons. First, they allow us to isolate the nonlinear streamwise velocity equation in order to demonstrate that its nonlinear coupling filters an appropriately constructed $\psi_{s s}(y, z)$ towards the expected "S" shape of the turbulent velocity profile. It also gives insight into mechanisms that create the momentum (energy) transfer, which generates this blunted profile. This relationship can be explored by noting that the forced solutions represent a balance between the dissipation $\frac{\Delta}{R} u_{s w}^{\prime}$ and a combination of the nonlinear terms, $\frac{\partial \psi}{\partial z} \frac{\partial u_{s w}^{\prime}}{\partial y}-\frac{\partial \psi}{\partial y} \frac{\partial u_{s w}^{\prime}}{\partial z}$, plus the mean shear term, $\frac{\partial \psi}{\partial z} \frac{\partial U}{\partial y}$. It is easy to show that the nonlinear terms are energy conserving. Thus, we are left with a balance between the dissipation and the streamwise constant part of the vortex tilting term and associated lift-up mechanism described by Landahl. ${ }^{29,30}$

The forced solutions shown herein are presented solely to demonstrate that the $2 \mathrm{D} / 3 \mathrm{C}$ model (Eq. (1)) is representative of certain aspects of turbulent behavior and as such, an amplification study based on this model is of interest. Gayme et al. $^{22}$ and Gayme ${ }^{27}$ provide a detailed exploration of the extent to which the 2D/3C (Eq. (1)) can be used as a model for turbulent behavior. The stability of the mean turbulent velocity profile for plane Couette flow in the face of threedimensional perturbations has previously been verified by Hwang and Cossu. ${ }^{31}$ The additional stability studies on the spanwise varying steady-state fields generated in this section require the analysis of a spatially distributed linear system with periodic coefficients, as described by Fardad and
Bamieh. $^{32}$ This requires extensive mathematical analysis that is beyond the scope of the current work and is currently under investigation.

For all of the results presented in this section, we solved for the forced solution $u_{s w_{s s}}^{\prime}(y, z)$ both using a least-squares approach and iteratively using an explicit Euler method for comparison. The initial studies were carried out using the same grid resolution as in the DNS data described by Tsukahara et al. ${ }^{24}$ (i.e. using a $96 \times 512$ grid on the $y-z$ plane). We then reduced the resolution to a $y-z$ plane grid of $48 \times 100$. We found negligible differences in the results between these two grid sizes. In the sequel, we only report the results for the $48 \times 100$ grid and the explicit Euler iterative solution.

\section{A. The velocity field}

Figure 2(a) shows a contour plot of $u_{s w_{s s}}^{\prime}(y, z)$ resulting from a stream function $\psi_{s s}$ with the same parameters as in Figure 1(a) (i.e., $q=1, \quad \varepsilon=0.00675$, and $\lambda_{z}=1.8$, at $R=3000$ ). This figure shows that our computed streamwise deviation from the laminar velocity has features consistent with the difference between a laminar and turbulent velocity field. For example, the velocity gradients near the walls are associated with an "S" shaped (or blunted) turbulent velocity profile. Figure 2(a) also shows good qualitative agreement with the $x$-averaged DNS data [shown in Figure 2(b) with the same contour levels as 2(a)]. This $x$-averaged DNS data have previously been shown to have a spanwise mean velocity corresponding to the full turbulent velocity profile. ${ }^{22}$ The steady-state streamwise velocity deviation from laminar $u_{s w_{s s}}^{\prime}(y, z)$ has similar structural features to the DNS data in that both have near-wall minimum and maximum peaks that are out of spanwise phase with one another top-to-bottom. There is, however, more coherence in our deviation from laminar across the span as compared to the DNS field, which is expected due to our use of a single harmonic in both the spanwise and wall-normal directions. 


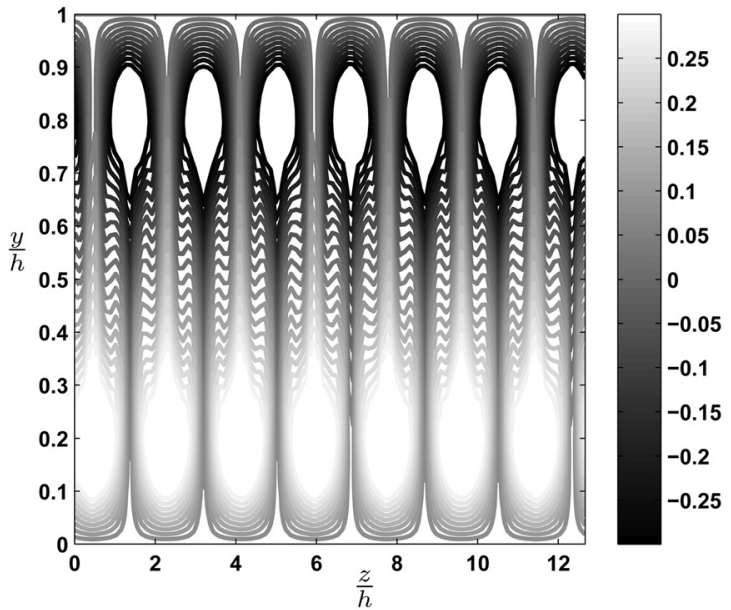

(a)

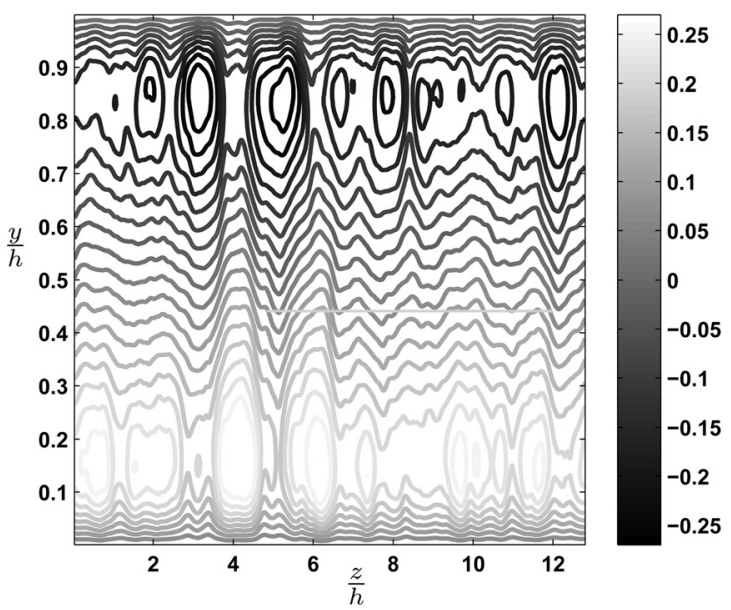

(b)

FIG. 2. Contour plots of (a) $u_{s w_{s s}}^{\prime}$, from $\psi_{s s}(y, z)=0.00675 \sin ^{2}(\pi y) \cos \left(\frac{2 \pi}{1.82} z\right)$ and (b) $u_{x_{\text {ave }}}^{\prime}$ the streamwise velocity component of the $x$-averaged DNS data. All plots correspond to $R=3000$ and have the same contour levels. These plots are reproduced from Ref. 22 .

\section{B. Mean profile}

The effective energy redistribution through the forced streamwise velocity deviations is further investigated through a spanwise mean over the streamwise velocity deviation from laminar. This is carried out at five perturbation amplitudes $(0.000675 \leq \varepsilon \leq 0.02)$, all at $R=3000$. Figure 3 shows averages across the span of $u_{s w_{s s}}^{\prime}(y, z)$ for these five $\varepsilon$ values along with a similar average of the $x$-averaged streamwise velocity field of the DNS. The spanwise average of the DNS has been validated against other results in the literature by Tsukahara et al. ${ }^{24}$

The use of $\psi_{s s}$ from Eq. (2) as an input to the steadystate Eq. (1a) produces streamwise velocity profiles whose shapes are consistent with the $x$-averaged DNS data. However, the peaks are located at different wall-normal positions. The fact that an exact match (with DNS data) for the wallnormal peak position is not obtained is not unexpected given the simplicity of the wall-normal variation in the steady-state model, as well as the streamwise constant and steady-state

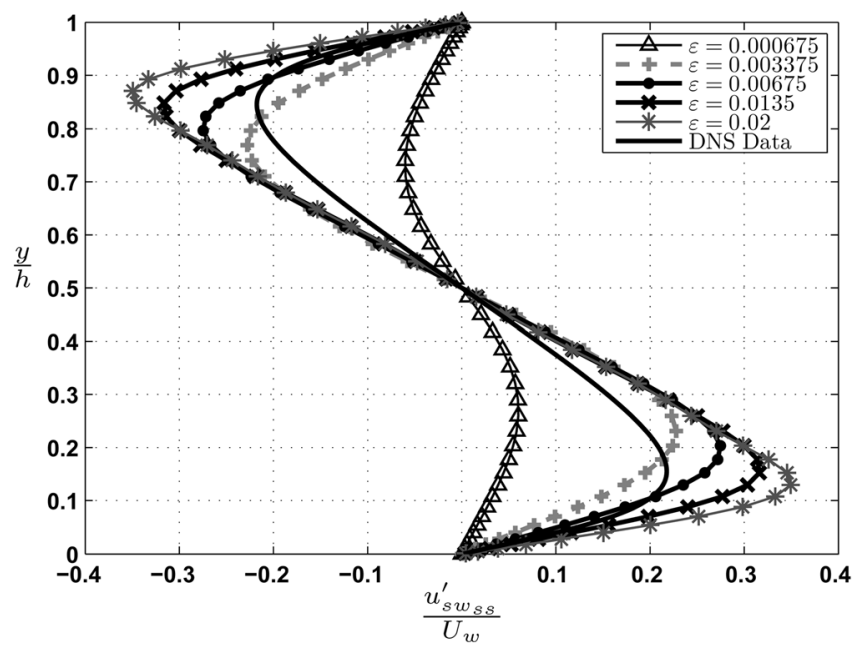

FIG. 3. Variation of the $2 \mathrm{D} / 3 \mathrm{C}$ (streamwise constant) deviation from laminar, $u_{s w_{c s}}^{\prime}$ with perturbation amplitude $(\varepsilon)$; based on input $\psi_{s s}(y, z)$ $=\varepsilon \sin ^{2}(\pi y) \cos \left(\frac{2 \pi}{1.82} z\right)$. A version of this figure is further discussed in Ref. 22. assumptions. Clearly, the full turbulent field is neither streamwise constant nor steady-state. The main point of presenting the velocity field arising from the forced steady-state model is to illustrate its effectiveness in reproducing the momentum redistribution associated with the change in the velocity profile from laminar to turbulent. This means that Eq. (1) provides more information about the turbulent velocity field than a linear model (which cannot produce the change in mean velocity profile between laminar and turbulent flows). Therefore, studying input-output amplification in this model may provide us with some additional insight compared to the traditional analysis performed using linear models.

The simple steady-state model described herein reasonably predicts the essence of the mean behavior at the expense of losing some of the smaller scale details. For example, an exact characterization of the wall-normal variation and, of course, the small-scale turbulent velocity fluctuations are not captured in this analysis. These results suggest that the phenomenon that is responsible for blunting of the velocity profile in the mean sense is a direct consequence of the interaction between rolling motions caused by the $y-z$ stream function and the laminar profile. In other words, this study provides strong evidence that the nonlinearity needed to generate the turbulent velocity profile is dominated by the nonlinear terms that are present in the $u_{s w}^{\prime}(y, z, t)$ evolution Eq. (1a).

\section{INPUT-OUTPUT AMPLIFICATION}

\section{A. Energy amplification}

In order to discuss input-output amplification, it is useful to determine the forcing required to produce a particular steady-state $\psi_{s s}$ described by Eq. (2). This is accomplished by solving a forced version of the steady-state $\psi$ evolution Eq. (1b) for the corresponding forcing term $\eta_{s s}(y, z)$, [note for $\eta_{s s}(y, z)=0$, i.e., the unforced equations, the stream function is zero]. We use a linearized version of Eq. (1b) because the nonlinear mechanisms that lead to the profile blunting are completely captured through the nonlinearity in Eq. (1b). ${ }^{22}$ 
The linearized version of this forcing, which by abuse of notation we also refer to as $\eta_{s s}(y, z)$, is given by

$$
\eta_{s s}(y, z)=-\frac{1}{R} \Delta^{2} \psi_{s s}
$$

This $\eta_{s s}$ can be viewed as the deterministic forcing required to produce a particular $\psi_{s s}$. In the sequel, we use the linear $\eta_{s s}$ of Eq. (3) for all of the computations. For a complete discussion of the use of a linear $\psi$ equation, see Ref. 27.

The input-output response can now be studied through an amplification factor of the form

$$
\Gamma_{s s}=\frac{\left\|u_{s w_{s s}}^{\prime}\right\|^{2}}{\left\|\eta_{s s}\right\|^{2}} .
$$

$\Gamma_{s s}$ is a nonlinear analog of the $L_{2}-$ to $-L_{2}$ induced norm that has been used to study the optimal response of the LNS with harmonic input/forcing, see, for example, Ref. 33. The energy in Eq. (4) is defined in terms of the squared 2-norm. For each two-dimensional component $\beta(y, z)$, this quantity is

$$
\begin{aligned}
\|\beta\|^{2} & =\int_{z_{1}}^{z_{2}} \int_{0}^{1} \beta(y, z)^{2} d y d z \\
& \approx \frac{\Delta z \Delta y}{L_{y} L_{z}} \sum_{k=1}^{N_{z}-1} \sum_{j=1}^{N_{y}-1} \beta^{2}\left(y_{j}, z_{k}\right),
\end{aligned}
$$

where $\Delta y=y_{2}-y_{1}$ and $\Delta z=z_{2}-z_{1}$ represent the space between the $y$ and $z$ grid points, respectively.

\section{B. Reynolds number scaling}

The scaling of $u_{s w_{s s}}^{\prime}$ with $R$ for a particular $\varepsilon$ is unclear from Eqs. (1a) and (2). An empirical relationship was computed using the stream function model (2) with $q=1$ and $\varepsilon=0.001$ for four different values of $R: 3000,6000,10000$, and 12000. Figure 4(a) shows that $\left\|u_{s w_{s s}}^{\prime}\right\|^{2}$ appears to scale as a function of $\sqrt{R}$ at the higher wave numbers $\left(k_{z}>2\right)$, for the $R$ values selected. The energy peaks also seem to collapse under this scaling for the higher Reynolds numbers we considered.

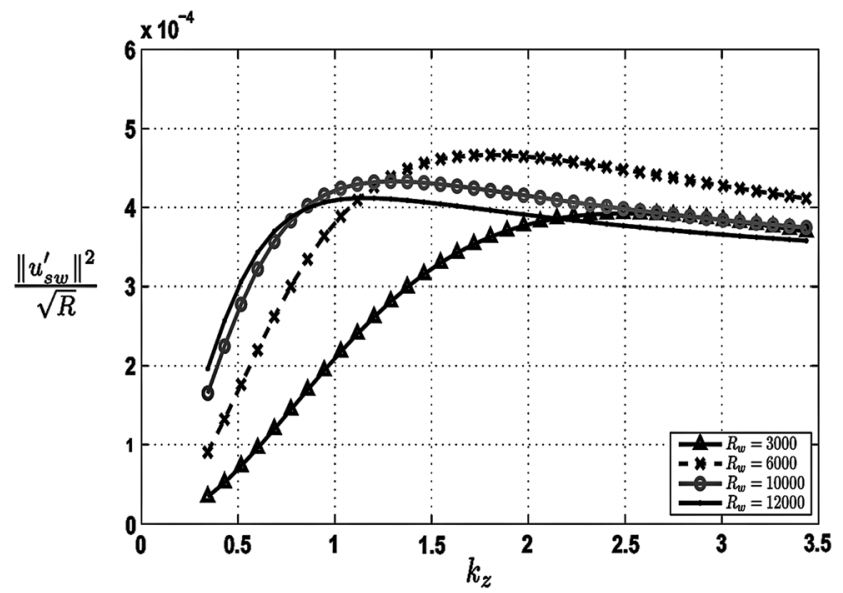

(a)

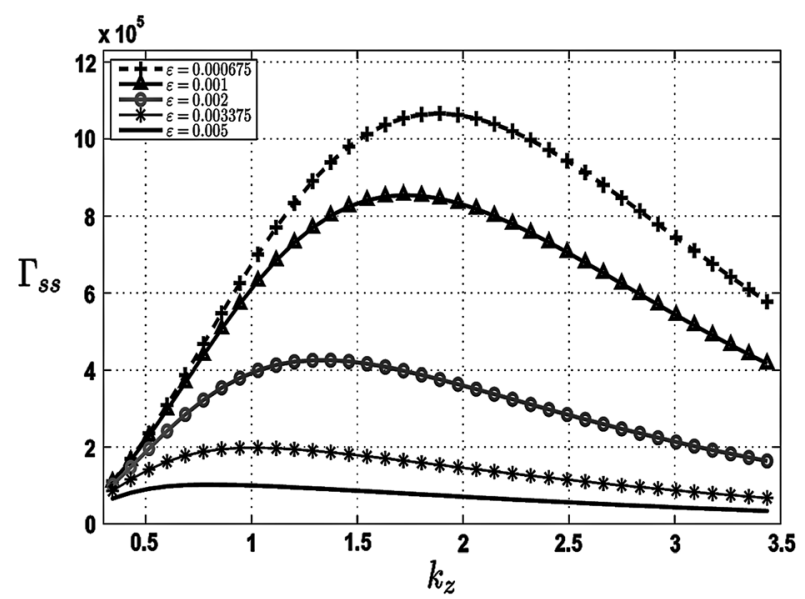

(c)

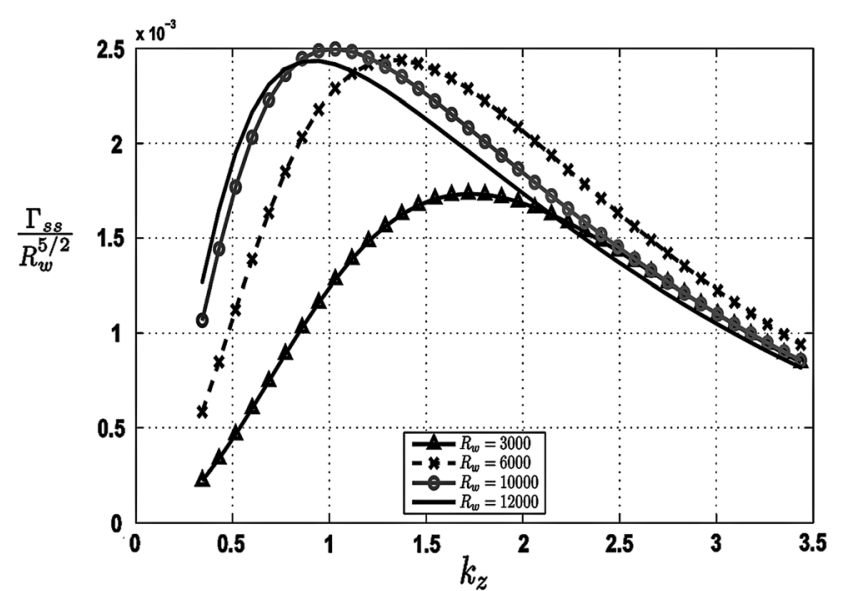

(b)

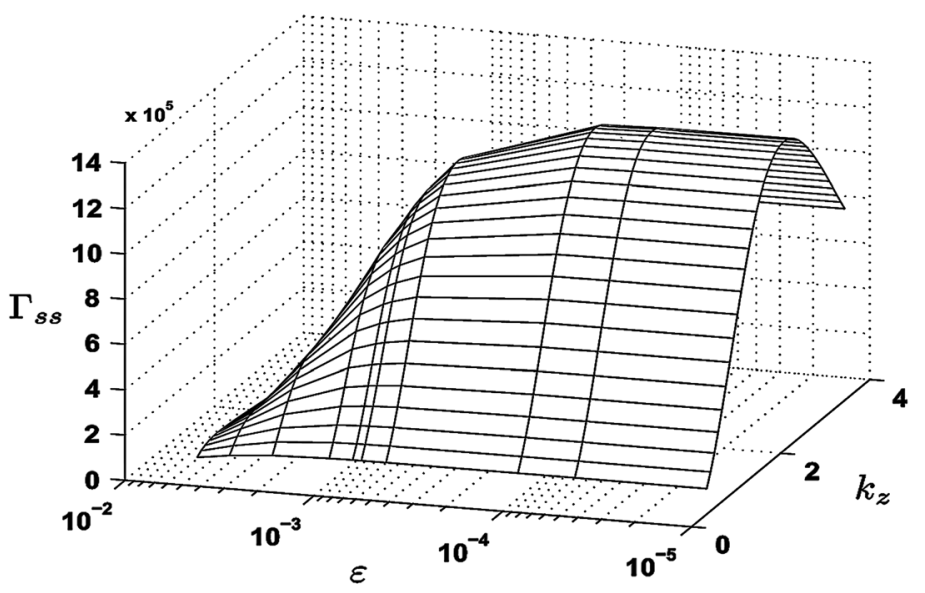

(d)

FIG. 4. (a) The streamwise energy scales as $\sqrt{R}$. (b) The amplification factor $\Gamma_{s s}$ scales as $R^{\frac{5}{2}}$. The optimal spanwise wavenumber occurs at the maximum $\Gamma_{s s}$ for each $R$. The change in peak response with increasing Reynolds number is interesting; however, the exact nature of the optimal input-output response is a function of both $k_{z}$ and $\varepsilon$. Therefore, one needs to find the corresponding optimal $\varepsilon$ in order to determine the true optimal spanwise large-scale feature spacing. (c) and (d) $\Gamma_{s s}$ for different values of $\varepsilon$ all at $R=3000$. Both $\Gamma_{s s}$ and the optimal spanwise wavenumber monotonically decrease with increasing $\varepsilon$. As $\varepsilon$ gets small, we approach the linearized equations because $u_{s w}^{\prime} \ll U$. Thus, linear mechanisms dominate for very small $\varepsilon$ and increasingly linearized equations have a larger input-output response. However, when $\varepsilon=0$, the forced solution (deviation from laminar) is zero and we recover the laminar solution. 
The scaling of $\Gamma_{s s}$ can be estimated by combining this $\sqrt{R}$ scaling of $\left\|u_{s w_{s s}}^{\prime}\right\|^{2}$ with the $\frac{1}{R}$ scaling of $\eta_{s s}(y, z)$, as seen in Eq. (3). Thus, $\Gamma_{s s}$ should scale as a function of $R^{\frac{5}{2}}$. Figure 4(b), which shows $\frac{\Gamma_{s s}}{R^{5 / 2}}$ for the same $R$ 's and $\varepsilon$, indicates that the amplification factor data do collapse well under the $R^{5 / 2}$ scaling, especially at the higher wave numbers. However, the $R=3000$ data peak does not seem to follow this relationship. This discrepancy can be explained by looking at previous linear studies.

The scaling of the input-output amplification for streamwise constant disturbances of the LNS equations was expressed as $f_{1}\left(k_{z}\right) R+f_{2}\left(k_{z}\right) R^{3}$ by Bamieh and Dahleh. ${ }^{2}$ Furthermore, they reported that the form of $f_{1}\left(k_{z}\right)$ and $f_{2}\left(k_{z}\right)$ means that the linear term dominates at low Reynolds numbers. ${ }^{2}$ The difference in scaling over different Reynolds number ranges was confirmed by a low Reynolds number linear study of Poiseuille flow that showed energy amplification at $k_{x}=0$ scales with $R^{\frac{3}{2}}$ for the range $800 \leq R \leq 5000$ and $R^{3}$ for larger Reynolds numbers. ${ }^{34}$ That study used a different characteristic length and velocity scale than in the present work and an equivalent normalization of our Reynolds number range yields $750 \leq R_{c} \leq 3000$, which is comparable to the range they considered. Optimal amplification studies based on initial conditions also support $R$ scaling at low Reynolds numbers. ${ }^{34}$ Based on these studies and the low Reynolds numbers employed in this study, it is reasonable that our scaling is less than $R^{3}$. The dominance of the linear scaling at low Reynolds numbers explains the lower $\Gamma_{s s}$ peak value for $R=3000$ (corresponding to $R_{c}=750$ based on the normalization used in Ref. 34).

\section{Optimal spanwise spacing}

Figure 4(a) indicates that $\left\|u_{s w_{s s}}^{\prime}\right\|^{2}$ increases with $k_{z}$ until it reaches a maximum value and then levels off. We can similarly find a relationship between $k_{z}$ and $\Gamma_{s s}$ by substituting the expression for $\psi_{\mathrm{ss}}$ from Eq. (2) into the linearized noise Eq. (3):

$$
\begin{aligned}
\eta_{s s}(y, z)= & \frac{\varepsilon}{R} \cos \left(k_{z} z\right)\left\{\left(8 q^{4} \pi^{4}+2 q^{2} k_{z}^{2} \pi^{2}\right)\right. \\
& \left.+\left[4 q^{2} k_{z}^{2} \pi^{2}-\left(k_{z}^{2}+4 q^{2} \pi^{2}\right)^{2}\right] \sin ^{2}(q \pi y)\right\} .
\end{aligned}
$$

Equation (6) illustrates that the noise scales with $k_{z}^{4}$ and $q^{4}$. So, the forcing energy $\left\|\eta_{s s}\right\|^{2}$ monotonically increases with $k_{z}$ while $\left\|u_{s w_{s s}}^{\prime}\right\|^{2}$ peaks and then levels off. Thus, even though larger $k_{z}$ is associated with higher forcing, the corresponding amplification factor does not continue to increase. There is an optimal $k_{z}$ that generates the most amplification: This is the dominant wavenumber corresponding to optimal spanwise spacing. In this section, we explore how changes in $\varepsilon$ and $R$ relate to the optimal spacing and discuss how our results compare to what has been previously reported in the literature.

The peak values of $\Gamma_{s s}$ for the Reynolds numbers considered in Figure 4(b) correspond to spanwise wave numbers of $k_{z}=0.86,1.0,1.4$, and 1.7 for $R=12000,10000,6000$, and 3000, respectively. This amounts to wavelengths of $7.3 h, 6.1 h, 4.6 h$, and $3.7 h$, respectively. To determine if these are optimal values over the entire parameter set, we need to determine the relationship between $\Gamma_{s s}$ and amplitude $\varepsilon$.

Figure 4(c) shows $\Gamma_{s s}$ for an amplitude range of $0.000675 \leq \varepsilon \leq 0.005$ all with $R=3000$. Over most of the range, both $\Gamma_{s s}$ and the optimal spanwise wavenumber monotonically decrease with increasing $\varepsilon$, although there appears to be a collapse at the minimal wavelengths. Therefore, the peak $\Gamma_{s s}$ over all the amplitudes we selected (i.e., the optimal $k_{z}$ ) occurs at the lowest $\varepsilon$. Figure 4(d) shows that continuing to reduce $\varepsilon$ results in convergence to an optimal wavenumber of $k_{z}=2.06$, which corresponds to $\lambda_{z}=3.05 h$, for all $\varepsilon \leq 0.0001$. We obtain the same optimal wavelength when we repeat this procedure for $R=6000,10000$, and 12000 .

Much of the literature (e.g., Refs. 1, 7, and 10) related to optimal spanwise spacing has shown optimal spanwise wave numbers $k_{z} \in[2.8,4]$. However, in many cases, these studies were aimed at determining spanwise spacing in the near-wall (inner-scaled) region. Recent Poiseuille flow studies using the LNS linearized about a turbulent velocity profile, where an eddy viscosity is used to maintain the profile, found that, at high Reynolds numbers, there are actually two peaks in the optimal energy growth curves, one scaling in inner units and the other in outer units. ${ }^{5,35}$ The outer unit peak appears to correspond to the large-scale structures that have a spanwise spacing of approximately $\lambda_{z} \in[2,5.2] \delta$ (where $\delta$ corresponds to the channel half height, i.e., $\frac{h}{2}$ ).

The only Couette flow study to look at both inner and outer unit scalings reported results at $R=3000 .{ }^{31}$ At this low Reynolds number, there is little to no scale separation between the peaks. They studied several input-output response functions and found that the optimal response to harmonic forcing occurs when $\lambda_{z}=3.85 h$. This harmonic forcing study is more closely related to our analysis than the initial condition-based studies reported in most of the other work. Couette flow DNS (Ref. 36) and experimental studies $^{26}$ have observed large-scale (outer region) feature spacing in the range $\lambda_{z}=[2,2.55] h$. Thus, our results $\left(\lambda_{z}=3.05 h\right)$ lie right in between those of the linear analytical Couette flow study $^{31}$ and the flow field observations. A constant optimal wavelength across Reynolds numbers is also consistent with previous studies using a linear model with an eddy viscosity based turbulent velocity profile. ${ }^{5,35}$

\section{Mean velocity profile versus optimal $\mathbf{k}_{\mathbf{z}}$}

Figure 5(a) shows the steady-state mean velocity profile computed using $\psi_{s s}$ models [Eq. (2)] with amplitudes in the range $0.001 \leq \varepsilon \leq 0.00675$ at their corresponding optimal values of $k_{z}$ along with the DNS data. All plots correspond to $R=3000$. The velocity gradients at the wall increase with $\varepsilon$. The fit in the center of the channel also approaches the DNS data as $\varepsilon$ increases, although, at $\varepsilon=0.005$ and above, the curve overshoots the DNS. There is no amplitude that exactly matches the DNS data and the fit is especially bad in the near-wall region. As previously discussed, this is because the assumptions inherent in the $2 \mathrm{D} / 3 \mathrm{C}$ model neglect the 


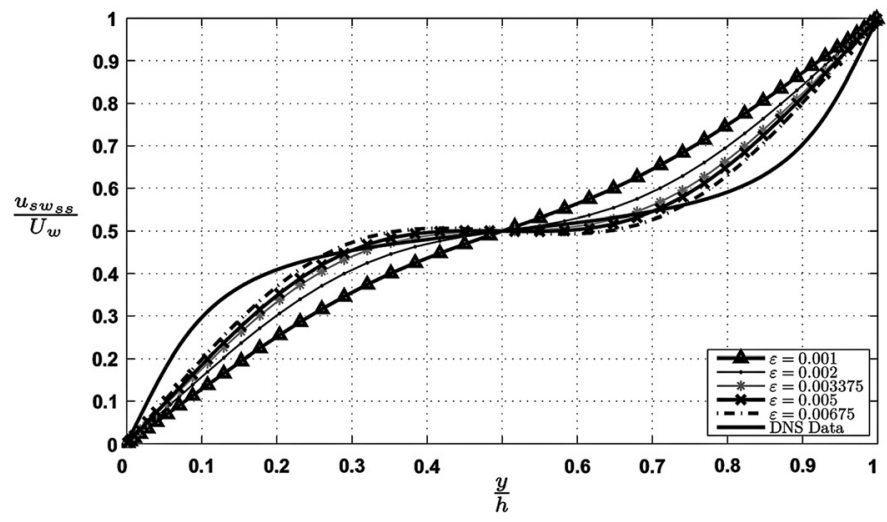

(a)

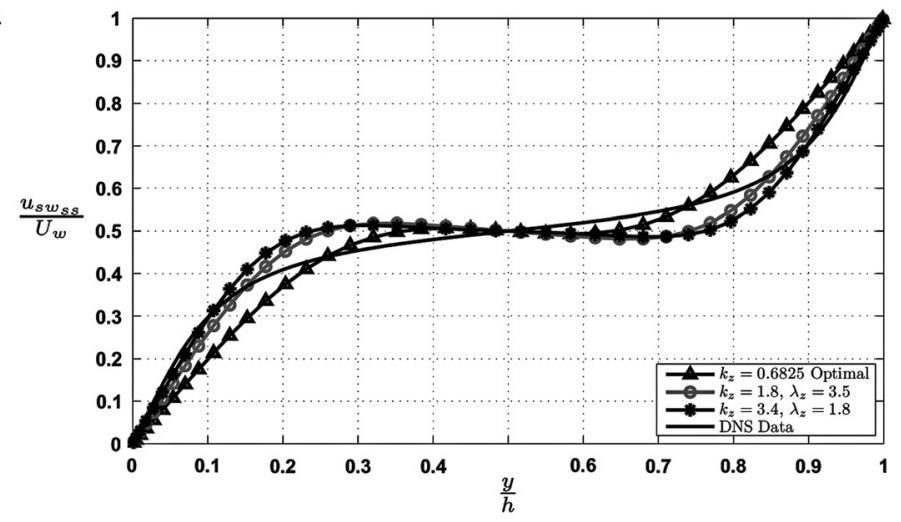

(b)

FIG. 5. (a) The mean velocity profile of the DNS data along with the one computed from the steady-state [Eq. (1a)] for a $\psi_{s s}$ model (Eq. (2)) with $q=1$, over a range of $\varepsilon$ with $k_{z}$ corresponding to the peak $\Gamma_{s s}$ for each $\varepsilon$ considered. (b) The mean velocity profile for $\varepsilon=0.00675$ at a number of different $k_{z}$ values compared with DNS data. The data in both (a) and (b) correspond to $R=3000$.

smaller scale activity that dominates in the near-wall region. In the core, the mean velocity curves, $\frac{u_{w_{w s}}}{U_{w}}$, corresponding to $\varepsilon=0.005$ and $\varepsilon=0.00675$, respectively, cross the DNS curve at a $y^{+} \approx 30$ and $y^{+} \approx 27$, based on the DNS viscous units. The maximum overshoot in the core (defined by $y^{+}>30$ in DNS viscous units) is $3.6 \%$ and $6.2 \%$, respectively, for $\varepsilon=0.005$ and $\varepsilon=0.00675$. This is remarkably good for such a simplified steady-state model.

The fact that the $\varepsilon=0.005$ and $\varepsilon=0.00675$ mean velocity profiles show the best agreement (most blunting) with the DNS data is not consistent with the fact that maximum amplification occurs at the smallest amplitudes (i.e., $\varepsilon \leq 0.0001)$. In order to study this further, we looked at different $k_{z}$ values corresponding to a $\psi_{s s}$ model amplitude of $\varepsilon=0.005$. Figure $5(\mathrm{~b})$ shows the mean velocity profile of the DNS along with mean velocities for $\varepsilon=0.005$ at the maximum $\Gamma_{s s}$ (optimal wavenumber $k_{z}=\frac{2 \pi}{\lambda_{z}}=0.69$ ), at $k_{z}=1.8$ and at $k_{z}=3.4$. This last value coincides with $\lambda_{z}=1.8$, i.e., the value corresponding to the dominant wavenumber of the $x$-averaged DNS data ${ }^{24}$ and the results discussed in Sec. III. Again, while by definition the amplitude of $\Gamma_{s s}$ is larger for the optimal wavenumber $k_{z}=0.6825$, the velocity profile has larger velocity gradients at the wall for higher values of $k_{z}$. This continued increase in shear stress at the wall as both $k_{z}$

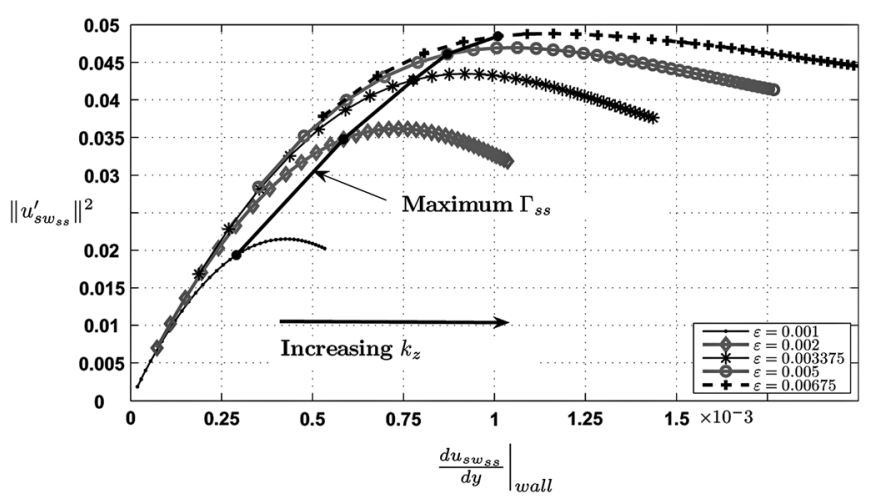

FIG. 6. The velocity gradient at the wall continues to increase while both $\Gamma_{s s}$ and the energy $\left\|u_{s w_{s s}}^{\prime}\right\|^{2}$ peak and then drop off. The solid black line represents the peak $\Gamma_{s s}$ for each $\varepsilon$. and $\varepsilon$ increase is better seen in Figure 6, which depicts the energy in $u_{s s}^{\prime}$ versus the shear stress at the wall.

Small $\psi_{s s}$ model (2) amplitudes, $\varepsilon$ 's, correspond to lesser nonlinear coupling between the equations (because $u_{s w}^{\prime} \ll U$ so that the equations approach the linearized model). On the other hand, at higher $\varepsilon$, the nonlinear terms have a larger magnitude because $\varepsilon$ directly multiplies each of the nonlinear terms. As $\varepsilon$ decreases, the energy amplification increases but the velocity profile blunting decreases (i.e., the profiles become increasingly laminar-like). In fact, in the limit as $\varepsilon \mapsto 0$, the laminar flow is recovered (i.e., $u_{s w}^{\prime}=0$ ). This behavior can be interpreted as follows: the amplification is dominated by linear mechanisms, whereas the blunting comes from nonlinear interactions. Moreover, there is some tradeoff between energy amplification and the creation of a turbulent-like blunted mean velocity profile. The observation that blunting continues to increase with wavenumbers beyond the energy optimal wavenumber as depicted in Figure 6 appears to indicate that the exact relationship depends on the spanwise wavenumber. This type of dependence is consistent with linear amplification theory. Further understanding of this tradeoff may provide important insight into the mechanisms associated with both transition and fully turbulent flow.

\section{CONCLUSIONS AND FUTURE WORK}

A simple cross-stream model of large-scale streamwise elongated structures nonlinearly coupled through a steadystate $2 \mathrm{D} / 3 \mathrm{C}$ streamwise momentum equation allows us to isolate important mechanisms involved in determining the shape of the turbulent velocity profile. The momentum redistribution that produces features consistent with the mean characteristics of fully developed turbulence appears to be directly related to the $2 \mathrm{D} / 3 \mathrm{C}$ nonlinear coupling in the streamwise velocity evolution Eq. (1a). The steady-state 2D/ 3C model produces a blunted, turbulent-like profile using very simple stream functions. This behavior appears to be robust to small changes in the stream function model. This repeatability suggests a preference for redistribution of momentum along the wall-normal direction. Further understanding of the underlying dynamics of this mechanism may 
provide insight into the transition problem and allow better design of turbulent suppression flow control algorithms.

An input-output analysis in this framework not only provides results consistent with previous studies but also illuminates an interesting interaction between energy amplification and the increased velocity gradient at the wall associated with the turbulent profile. Essentially, although the inputoutput amplification monotonically decreases with increasing forcing amplitude, the velocity profiles become increasingly more blunted. Thus, there is likely a tradeoff between the linear amplification mechanisms and nonlinear blunting mechanisms that determine important features of the turbulence-like phenomena modeled by Eq. (1). This tradeoff may have important implications for flow control techniques that target skin friction or the mean profile.

A natural extension of this work would be to refine our stream function model through adding additional wall-normal and spanwise harmonics to the existing form of Eq. (2). Developing an entirely new model using models of real sources of flow disturbances, as discussed in Ref. 22, may also provide guidance in determining a better stream function $\psi_{s s}$ as a forced steady-state solution of Eq. (1b).

\section{ACKNOWLEDGMENTS}

The authors would like to thank H. Kawamura and T. Tsukahara for providing them with their DNS data. This research is partially supported by AFOSR (FA9550-08-10043). B.J.M. gratefully acknowledges support from NSFCAREER Award No. 0747672 (program managers W. W. Schultz and H. H. Winter).

${ }^{1}$ B. F. Farrell and P. J. Ioannou, "Stochastic forcing of the linearized Navier-Stokes equations," Phys. Fluids A 5, 2600 (1993).

${ }^{2}$ B. Bamieh and M. Dahleh, "Energy amplification in channel flows with stochastic excitation," Phys. Fluids 13, 3258 (2001).

${ }^{3}$ M. R. Jovanović and B. Bamieh, "The spatio-temporal impulse response of the linearized Navier-Stokes equations," in Proceedings of the American Control Conference, Arlington, VA (2001), pp. 1948-1953.

${ }^{4} \mathrm{M}$. R. Jovanović and B. Bamieh, "Componentwise energy amplification in channel flows," J. Fluid Mech. 534, 145 (2005).

${ }^{5}$ J. C. del Álamo and J. Jiménez, "Linear energy amplification in turbulent channels," J. Fluid Mech. 559, 205 (2006).

${ }^{6} \mathrm{~B}$. J. McKeon and A. S. Sharma, "A critical-layer framework for turbulent pipe flow,” J. Fluid Mech. 658, 336 (2010).

${ }^{7}$ K. M. Butler and B. F. Farrell, "Three-dimensional optimal perturbations in viscous shear flow," Phys. Fluids A 4, 1637 (1992).

${ }^{8}$ B. F. Farrell and P. J. Ioannou, "Optimal excitation of three-dimensional perturbations in viscous constant shear flow," Phys. Fluids A 5, 1390 (1993).

${ }^{9}$ M. R. Jovanović and B. Bamieh, "Unstable modes versus non-normal modes in supercritical channel flows," in Proceedings of the American Control Conference, Boston, MA (2004), pp. 2245-2250.

${ }^{10} \mathrm{~L}$. H. Gustavsson, "Energy growth of three-dimensional disturbances in plane Poiseuille flow," J. Fluid Mech. 224, 241 (1991).

${ }^{11}$ M. J. Lee and J. Kim, "The structure of turbulence in a simulated plane Couette flow," Thin Solid Films 1, 531 (1991).
${ }^{12}$ K. H. Bech, N. Tillmark, P. H. Alfredsson, and H. I. Andersson, "An investigation of turbulent plane Couette flow at low Reynolds numbers," J. Fluid Mech. 286, 291 (1995).

${ }^{13}$ K. J. Kim and R. J. Adrian, "Very large scale motion in the outer layer," Phys. Fluids 11, 417 (1999).

${ }^{14}$ J. F. Morrison, B. J. McKeon, W. Jiang, and A. J. Smits, "Scaling of the streamwise velocity component in turbulent pipe flow," J. Fluid Mech. 1508, 99 (2004).

${ }^{15}$ M. Guala, S. E. Hommema, and R. J. Adrian, "Large-scale and very-largescale motions in turbulent pipe flow," J. Fluid Mech. 554, 521 (2006).

${ }^{16} \mathrm{~N}$. Hutchins and I. Marusic, "Evidence of very long meandering structures in the logarithmic region of turbulent boundary layers," J. Fluid Mech. 579, 1 (2007).

${ }^{17}$ N. Hutchins and I. Marusic, "Large-scale influences in near-wall turbulence," Philos. Trans. R. Soc. London A 365, 647 (2007).

${ }^{18} \mathrm{~F}$. Waleffe, "Proposal for a self-sustaining mechanism in shear flows," Tech. Rep., Center for Turbulence Research, Stanford University/NASA Ames, 1990.

${ }^{19}$ J. M. Hamilton, J. Kim, and F. Waleffe, "Regeneration mechanisms of near-wall turbulence structures," J. Fluid Mech. 287, 317 (1995).

${ }^{20} \mathrm{~F}$. Waleffe, "On a self-sustaining process in shear flows," Phys. Fluids $\mathbf{9}$, 883 (1997).

${ }^{21}$ J. Jiménez and A. Pinelli, "The autonomous cycle of near-wall turbulence,” J. Fluid Mech. 389, 335 (1999).

${ }^{22}$ D. F. Gayme, B. J. McKeon, A. Papachristodoulou, B. Bamieh, and J. C. Doyle, "Streamwise constant model of turbulence in plane Couette flow," J. Fluid Mech. 665, 99 (2010).

${ }^{23}$ D. F. Gayme, B. McKeon, A. Papachristodoulou, and J. C. Doyle, “ 2D/3C model of large scale structures in turbulence in plane Couette flow," in Proceedings of the 6th International Symposium on Turbulence and Shear Flow Phenomenon, Vol. I, Seoul, Korea (2009), pp. 283-288.

${ }^{24}$ T. Tsukahara, H. Kawamura, and K. Shingai, "DNS of turbulent Couette flow with emphasis on the large-scale structure in the core region," J. Turbulence, 7 (2006).

${ }^{25}$ K. M. Bobba, "Robust flow stability: Theory, computations and experiments in near wall turbulence," Ph.D. thesis (California Institute of Technology, Pasadena, CA, 2004).

${ }^{26} \mathrm{O}$. Kitoh and M. Umeki, "Experimental study on large-scale streak structure in the core region of turbulent plane Couette flow," Phys. Fluids 20, 025107 (2008).

${ }^{27}$ D. F. Gayme, "A robust control approach to understanding nonlinear mechanisms in shear flow turbulence," Ph.D. thesis (California Institute of Technology, Pasadena, CA, 2010).

${ }^{28}$ D. Barkley and L. S. Tuckerman, "Mean flow of turbulent-laminar patterns in plane Couette flow," J. Fluid Mech. 576, 109 (2007).

${ }^{29}$ M. T. Landahl, "Wave breakdown and turbulence," SIAM J. Appl. Math. 28, 735 (1975).

${ }^{30}$ M. T. Landahl, "A note on an algebraic instability of inviscid parallel shear flows,” J. Fluid Mech. 92, 243 (1980).

${ }^{31}$ Y. Hwang and C. Cossu, "Amplification of coherent streaks in the turbulent Couette flow: an input-output analysis at low Reynolds number," J. Fluid Mech. 643, 333 (2010).

${ }^{32}$ M. Fardad and B. Bamieh, "Perturbation methods in stability and norm analysis of spatially periodic systems," SIAM J. Control Optim. 47, 997 (2008).

${ }^{33}$ P. J. Schmid and D. S. Henningson, Stability and Transition in Shear Flows, Applied Mathematical Sciences Vol. 142 (Springer-Verlag, New York, 2001).

${ }^{34}$ B. F. Farrell and P. J. Ioannou, "Variance maintained by stochastic forcing of non-normal dynamical systems associated with linearly stable shear flows," Phys. Rev. Lett. 72, 1188 (1994).

${ }^{35}$ G. Pujals, M. García-Villalba, C. Cossu, and S. Depardon, "A note on optimal transient growth in turbulent channel flows," Phys. Fluids 21, 015109 (2009).

${ }^{36}$ J. Komminaho, A. Lundbladh, and A. V. Johansson, "Very large structures in plane turbulent Couette flow," J. Fluid Mech. 320, 259 (1996). 